\title{
Trauma focused cognitive behavioral group therapy for abused children with post traumatic stress: the case of institutionalized children, addis ababa, ethiopia
}

\begin{abstract}
Introduction: Sexual and physical abuse against children and adolescents are major public health and social problems around the world. Child physical and sexual abuses occur in all cultural, socio economic, educational, racial and ethnic groups. The high prevalence and the devastating negative consequences of sexual and physical abuse initiate research inquiry in the intervention and treatment aspects of the problem.

Objective: This study examined whether Trauma Focused Cognitive Behavioral Group Therapy (TF-CBGT) is effective in treating both sexually and physically abused children among Kechene and OPRIFS (Organization for Prevention, Rehabilitation and Integration of Female Street Children) Children's home.

Methods: It is a control group's pretest-posttest quasi-experimental design. Sixty female participants aged 8 to 18 were selected purposefully. So as to make equivalent in terms of their score of child post traumatic stress symptoms scale and types of abuse they experienced, participants were divided in to treatment and control group. The treatment group received TF-CBGT for 3 sessions a week for a total of 12 contacts. Child posttraumatic stress disorder symptoms scale (CPSS) used to measure post traumatic stress symptoms of participants. Test was conducted before the treatment, just after the treatment and 15 days after the treatment.

Results: TF-CBGT is potentially effective in reducing symptoms of posttraumatic stress disorder from pretest to posttest measures. However, it is recommended to conduct a large scale study with more sample size and diversity to expand the findings of this study.
\end{abstract}

Volume 2 Issue 4 - 2015

\author{
Riyadh Abdella, Daniel Sewasew, Gebeyehu \\ Abate, Mastewal Bitew \\ Psychology Department, University of Gondar, Ethiopia
}

Correspondence: Daniel Sewasew, Postal box 196, Gondar. Ethiopia,Tel 25I-910116009, Email danielsew@yahoo.com

Received: January 23, 2015 | Published: April 07, 2015

Keywords: posttraumatic stress, tf-cbgt, sexual abuse, physical abuse

Abbreviations: TF-CBGT, trauma focused cognitive behavioral group therapy; OPRIFS, organization for prevention, rehabilitation and integration of female street children; CPSS, child posttraumatic stress disorder symptoms scale; UN, united nations; PTSD, post traumatic stress disorder; CPTSS, child post traumatic symptoms scale

\section{Introduction}

Sexual and physical abuse against children and adolescents are major public health and social problems around the world. According to world health organization estimates, $20 \%$ of females and 5 to $10 \%$ of males in the general population reported child sexual abuse. ${ }^{1}$ The overall prevalence of physical abuse is estimated to be 10 to $25 \%$ in the general population. Child physical and sexual abuses occur in all cultural, socio economic, educational, racial and ethnic groups. This kind of violence may cause a number of adverse effects in the victim's physical, cognitive, emotional and social development. The problems and symptoms that have been associated repeatedly with childhood sexual abuse and physical abuse history are often over lapping. Among these overlapping symptoms the most common are symptoms of posttraumatic stress disorder, low self-esteem and guilt, anxiety, depression and interpersonal dysfunction. ${ }^{2}$

Child abuse or child maltreatment includes all forms of physical and emotional ill treatment that results in actual or potential harm to the child's health, development or dignity. ${ }^{3}$ This broad definition includes sexual abuse, physical abuse, emotional abuse, neglect and negligent treatment and child exploitation. Among these sexual abuse and physical abuse are the most disastrous and well studied. ${ }^{4}$ Sexual abuse is defined as sexual activity with a child or adolescent that he/she does not fully understand, to which he/she is incapable of consenting, or that violets social taboos. ${ }^{5}$ Child physical abuse refers to deliberately causing physical damage or pain on a child. The physical injury may result from many different acts including hitting, kicking, slapping, burning choking, throwing, whipping and/or padding. ${ }^{2}$

As mentioned above both sexual abuse and physical abuse are frequently associated with various types of psychological or mental disorders. However, PTSD is assumed as the best conceptualization of disturbance that occurs after child abuse in many researches. Studies have shown that almost half of sexual abuse survivors will develop PTSD symptoms. ${ }^{6}$ It has been suggested that, this is due to the frequent presence of various traumatizing factors during or after sexual abuse like interpersonal violence, physical injury and fear of dying. Many studies also indicate that physical abuse frequently leads to post traumatic stress disorder since it involves both physical and psychological trauma as a result of sudden and brutal attack on a child who cannot defend him/her self.?

Various efforts have been made worldwide to prevent or reduce child sexual and physical abuse. United Nations (UN) convention 
on child rights insures children's rights of provision of services, participation in society and rights of protection and care. The role of criminal justice system is significant in discouraging the perpetrators behavior, reinforcing the low and providing relief for the victims. Efforts are also being made by advocates to create public awareness regarding the child maltreatment. Various programs are developed and implemented in different countries to prevent or minimize child abuse. Researchers have made considerable contributions to understand various aspects of the issue, like the prevalence, causes and consequences of violence against children.

As far as intervention is concerned understanding the nature and extent of abuse and neglect and their consequences is important to the design of treatment programs. Many treatment programs and models have been developed to specifically deal with the psychological problems of children who have experienced sexual as well as physical abuse. Some of them include abuse focused cognitive behavioral therapy, play therapy, music therapy, art therapy, non directive supportive therapy, developmental touch therapy and trauma focused cognitive behavioral therapy. However, the effectiveness of these therapy modalities is yet to be investigated.

The high prevalence of sexual and physical abuse and the negative psychological consequences initiate researchers to investigate the effectiveness of psychotherapy in treating victims. One Meta analysis has summarized various research findings on psychotherapy of abused children. ${ }^{8}$ This study has concluded that cognitive behavioral therapy was relatively more effective than other treatment modalities, and recommended more researches and replications with more rigorous research designs. In addition, it indicated that trauma focused cognitive behavioral therapy (TF-CBT) was more effective in reducing symptoms of post traumatic stress disorder (PTSD) and restructuring dysfunctional beliefs which accompany various psychological disorders.

The field of empirical research into the psychological treatment of children who have been sexually and/or physically abused is relatively new, only few studies can be mentioned before1980. In Ethiopia few research works have been carried out about child sexual and/or physical abuse in general not to mention the treatment aspect in particular. More recent research worldwide has tended to focus on cognitive behavioral therapy ${ }^{9}$. Important questions remain about the efficacy and effectiveness of different psychological treatments in Ethiopian context. The aim of this study is to assess the effectiveness of cognitive behavioral group therapy in the treatment of post traumatic stress symptom of sexually or physically abused children.

Studies conducted in Ethiopia indicate that there is high prevalence of child abuse. For instance, in one study $38.5 \%$ of the sample who are students reported that they have experienced sexual abuse at least once. ${ }^{10}$ Physical punishment (corporal punishment) of children is culturally accepted which increase the incidence of physical abuse in our culture. The negative consequences of child physical and sexual abuse can get even worse (exacerbated) by wide spread poverty and HIV Aids. Ethiopia has ratified the convention on the rights of the child which entitles children protection from all forms of abuse until age 18. The criminal code of Ethiopia has a number of protective provisions against child abuse. In addition to the legal provision, various efforts have also been made to raise public awareness on the issue of child abuse.

However, little or no efforts have been made to address the psychological needs of the victims of child abuse. More importantly, it is not known to what extent the treatment approaches developed in western countries work to Ethiopian cultural context. Research in USA, Europe and other parts of the world indicate that cognitive behavioral therapy is one of the most empirically supported therapy technique among the many therapy techniques that are developed to deal with the needs of abused children. ${ }^{11}$ However, adequate efforts have not been made to adopt or investigate the effectiveness of cognitive behavioral therapy in Ethiopian context.

Based on the above discussion, the research attempted to answer the fallowing research questions:

i. Is there statistically significant difference in posttraumatic stress symptoms from pre-to-post treatment as well as from post treatment to follow up measures in the treatment group (abused children who were treated with trauma focused cognitive behavioral therapy)?

ii. Is there a statistically significant mean difference in posttraumatic stress symptoms from pre-to-post treatment measures between treatment group and control group?

iii. Is there statistically significant difference between the post traumatic stress symptoms score of the children who experienced physical abuse and those who experienced sexual abuse and younger children ( 9 to 12 years of age) and the teenagers (13 to 16years of age)at pre test and post test one?

\section{Materials and methods}

\section{Study design}

The study was a quasi experimental control group pre-post design with intra and inter-group comparisons (Table 1). The intervention was carried out within treatment groups whereas the outcome compared within the same groups as well as between the treatment and control/comparison groups. Participants were assessed in three different stages: before group therapy, just after the group therapy and 15 days after therapy.

Table I Quasi experimental design

\begin{tabular}{llll}
\hline $\begin{array}{l}\text { Study } \\
\text { Groups }\end{array}$ & $\begin{array}{l}\text { First } \\
\text { Observation }\end{array}$ & Treatment & $\begin{array}{l}\text { Second } \\
\text { Observation }\end{array}$ \\
\hline $\begin{array}{l}\text { Treatment } \\
\text { Group }\end{array}$ & O1 & $\sqrt{ }$ & O2 \\
\hline $\begin{array}{l}\text { Control } \\
\text { Group }\end{array}$ & OI & $X$ & O2 \\
\hline
\end{tabular}

\section{Research site}

The study was conducted in Kechene and OPRIFS children's home. These are nongovernmental charity organizations, where; Kechene children's home was established in 1945 while OPRIFS was established in 2008. Kechene children's home currently accommodates 233 girls up to 18years of age while OPRIFS accommodate 190 children enrolling for rehabilitation services. Most children in Kechene children's home stay up to 18 years of age some of them integrated with their family while OPRIFS usually provides temporary shelter for children until they integrate with their families.

\section{Population and sample}

From both charity organizations (Kechene children's home and OPRIFS), 60 female children were selected as participants based on the following criteria: 
1. They have experienced either sexual or physical abuse

2. They are within the age range of $8-18$

3. They are willing to participate in the study

\section{They scored more than 15 on the CPSS}

They were identified by the social workers and counselors who are working with the children. All the participants scored more than 15 on the CPSS which suggest that they at least show mild symptoms of PTSD. The detailed profile of the subjects presented below.

\section{Demographic characteristics of treatment and control groups}

The majority of participants age range was 13-16 (63.3\%) for treatment group's whereas $53.3 \%$ were control groups. Likewise, $73 \%$ of treatment groups and $83 \%$ of control groups were elementary school students. There were equal number of physically and sexually abused children in both treatment and control groups. Out of the total participants, $86.3 \%$ of treatment groups and $92.6 \%$ of control group stayed less than a month in the institutions. All participants in both groups and institution have never been received psychotherapeutic service prior to the study.

\section{Allocation of participants to treatment and control group}

After the participants take the pre test measures of child post traumatic symptoms scale (CPTSS), they were divided in to two groups. The participants were allocated to one of the groups purposefully based on their pre test score and type of abuse they experienced. Then, lottery method was used to determine which group would be treatment group (the group that receives treatment) and control group (the group that is used as a comparison only).

\section{Instrument}

Two sets of questionnaires (demographic data sheet and Standardized test) were used to gather the required information from the participants before and after the intervention. Demographic data sheet was used to gather demographic information whereas standardized test i.e. child posttraumatic stress symptoms scale was used to measure post traumatic stress symptoms.

\section{The child post traumatic stress symptoms scale (CPSS)}

It was used to measure children's post traumatic stress level after they have experienced either physical or sexual abuse. The children's post traumatic stress level was measured before treatment, just after the treatment and 15 days after treatment. However, the control groups were measured at two points in time which is before the treatment and just after the treatment.

The child post traumatic stress symptoms scale (CPSS), which is used for children aged 8 to 18 , is based on diagnostic statistical manual version four (DSM-IV) criteria of post traumatic stress disorder. ${ }^{12}$ It has two parts. Part one is consisted of 17 items, each item representing PTSD symptom as described by DSM-IV. These items are rated based on likert scale ranging from 0 (no symptom in two weeks) to 3(five or more times in two weeks) yielding a total score which ranges from 0-51. The second part is consisted of 7 items which measures levels of functional impairment as a result of the symptoms of PTSD. The second part is rated dichotomously as absent (0) or present (1) yielding a total score range from 0 -7. Higher score indicating greater functional impairment. Hence, a total score for both parts ranges from 0 to 58 .

Psychometrically, the test is characterized as .84 internal consistencies and its convergent validity was also high that its correlation with child post traumatic stress index is $0.80 . .^{12}$ The total score of CPSS ranges from 0 to 58. Taking 15 as a cutoff score, the following ranges were adapted for the purpose of this study: ${ }^{12}$ Scores between 0 and 15 are indicative of minimum levels of posttraumatic stress symptoms.

a. Scores between 16 and 24 are indicative of mild levels of posttraumatic stress symptoms.

b. Scores between 25 and 39 are indicative of moderate levels of posttraumatic stress symptoms.

c. Scores between 40 and 58 are indicative of severe levels of posttraumatic stress symptoms.

\section{Translation and pilot testing}

The standardized child post traumatic stress scale (CPTSS) was translated earlier by other researchers and adopted for this study. It was pilot tested on 20 samples to test its reliability. The Cronbach alpha's inter item correlation coefficient was 0.73 . However, due to the fact that younger children needed some support to understand the items, it was administered in the form of interview by the researcher.

\section{Ethical considerations}

Throughout the research process, all ethical guidelines and principles were considered. Participants' informed consent (willingness) was the basis to participate in testing and the treatment program. The participants were assured that whatever they share in the process of the study (in counseling and in testing results) would be strictly confidential.

\section{Data analysis}

SPSS version 16 (the statistical package for social science) were used to analyze the quantitative data. Moreover, descriptive statistics, frequency distributions and percentage were used to describe participants' demographic characteristics. Dependent and independent t-test is used to compare the mean difference between the pre-test and post-test as well as follow up measures of CPSS.

\section{Results}

\section{Post traumatic stress symptoms (PTSS)}

As shown on the Table 2 before the treatment $56.7 \%$ of participants in the treatment group were on moderate level of post traumatic stress symptoms, $36.7 \%$ showed mild level of PTSD symptoms, while a $6.7 \%$ measured high on the scale. After the treatment those on the moderate level decreased to $46.7 \%$, those on the mild level of post traumatic stress symptoms increased to $30 \%$ and there were no respondent on the sever level of PTSD symptoms. These treatment gains at post test 1 (just after the treatments) were maintained at post test 2 (follow up) measures.

Table 2 also show that, $60 \%$ of participants in the control group have shown moderate level of post traumatic stress symptom, $26.7 \%$ mild and the rest $3.3 \%$ showed sever level of PTSD symptoms. At post treatment level of measurement $60 \%$ showed moderate level of PTSD, $40 \%$ were mild and no one was measured sever on the scale. 


\section{Treatment outcome within treatment and control groups}

Table 3 indicates that the mean post traumatic stress symptoms score for the treatment group before treatment was 27.13, with $S D$ of 6.58. After the treatment (post test 1) the mean post traumatic stress symptoms score was reduced to 24.70 with $S D$ of 5.91 the mean difference being 2.43. Paired test indicated that the difference is statistically significant $(d f=29, t=2.613)$.

It is shown on the Table 3 above that, the mean post traumatic stress symptoms measure for the control group at the first point of measurement (pre test) was 28 with standard deviation of 4.92. At the second point of measurement (post test) it was reduced to 27.56 with standard deviation of 4.76.The difference of the two measures was 0.4333 which indicates a reduction in the level of symptoms. However, the difference was not statistically significant.

As Table 4 indicates the mean post traumatic stress symptoms score just after the treatment was 24.70 with a SD of 5.91 while it was 25.13 with SD of 6.27 at the follow up measure (post test two). The difference between the two means was 0.4333 which is not statistically significant.

Table 2 Posttraumatic stress symptom level of treatment group and control group before treatment after treatment and at follow up

\begin{tabular}{lllllll}
\hline Level of PTSS & Pre Test & \multicolumn{3}{l}{ Post Test I } & \multicolumn{2}{l}{ Post Test 2 } \\
\hline Treatment Group & $\mathrm{F}$ & $\mathrm{P}(\%)$ & $\mathrm{F}$ & $\mathrm{P}(\%)$ & $\mathrm{F}$ & $(\%)$ \\
0-15 (Minimum) & & & & & & \\
16-24 (Mild) & $\mathrm{II}$ & 36.7 & 16 & 53.3 & 16 & 53.3 \\
25-39 (Moderate) & 17 & 56.7 & 14 & 46.7 & 14 & 46.7 \\
40-58 (Severe) & 2 & 6.7 & - & - & - & - \\
Total & 30 & 100 & 30 & 100 & 30 & 100 \\
Control Group & $\mathrm{F}$ & $\mathrm{P}(\%)$ & $\mathrm{F}$ & $\mathrm{P}(\%)$ & $\mathrm{F}$ & $(\%)$ \\
0-15 (Minimum) & & & & & & \\
16-24 (Mild) & 10 & 33.3 & 12 & 40 & & \\
25-39 (Moderate) & 18 & 60 & 18 & 60 & & \\
40-58 (Sever) & 2 & 6.7 & - & - & & \\
Total & 30 & 100 & 30 & 100 & & \\
\hline
\end{tabular}

*Note: F (Frequency) P (Percentage)

Table 3 Dependant t-test of the mean post traumatic stress scores of the treatment and control group before and after the treatment $(n=30)$

\begin{tabular}{lllll}
\hline Group & \multicolumn{5}{l}{ Post traumatic Stress } & Symptoms Scores \\
\hline Treatment Group & Mean & SD & t & Sig \\
Before Treatment (Pre Test) & 27.13 & 6.58 & 2.618 & 0.014 \\
After Treatment (Post Test) & 24.7 & 5.91 & & \\
Paired Difference & 2.43 & & & \\
Control Group & Mean & SD & t & Sig \\
Before Treatment (Pre Test) & 28 & 4.92 & 0.433 & 0.668 \\
After Treatment (Post Test) & 27.56 & 4.76 & & \\
Paired Difference & 0.43 & & & \\
\hline
\end{tabular}

*Statistically significant at $\mathrm{P}<.05$

Table 4 Paired t-test of the mean post traumatic stress scores of the treatment group at post test one and post test two $(n=30)$

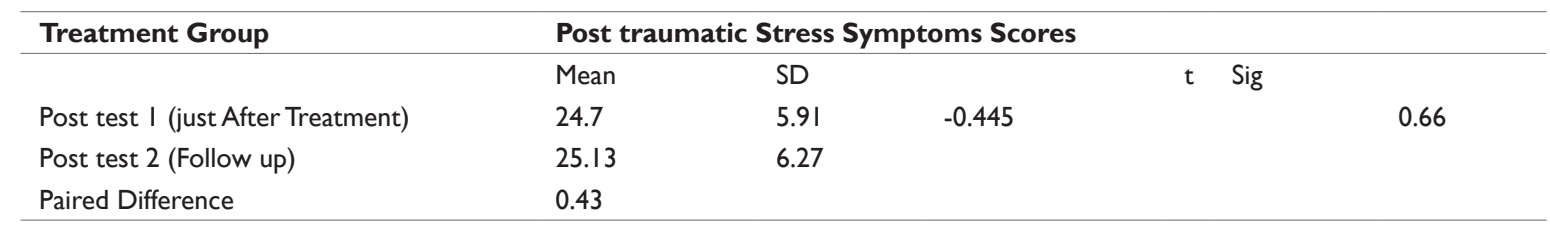

*Statistically significant at $\mathrm{P}<.05$

Table 5 Independent t-test of the mean post traumatic stress scores of the treatment group and control group

\begin{tabular}{llllll}
\hline PTSD Score & Groups & & & & \\
\cline { 2 - 6 } & Treatment & Control & Mean Difference & t & Sig. \\
\hline Pre-test & 27.13 & 28 & 0.86 & -577 & 0.566 \\
Post-test & 24.7 & 27.56 & 2.3 & 2.068 & 0.043 \\
Mean Difference & 2.43 & 0.43 & & & \\
\hline
\end{tabular}

*Statistically significant at $\mathrm{P}<.05$ 
Table 6 Independent t-test of the mean post traumatic stress scores of the sexually abused and physically abused children

\begin{tabular}{llllll}
\hline PTSD Score & Groups & & & \\
\cline { 2 - 6 } & Sexually Abused & Physically Abused & Mean Difference & t & Sig. \\
\hline Pre-test & 28.07 & 26.41 & 1.66 & 0.68 & 0.502 \\
Post-test & 24.88 & 24.46 & 0.42 & -190 & 0.149 \\
Mean Difference & 3.19 & 2.88 & & & \\
\hline
\end{tabular}

*Statistically significant at $\mathrm{P}<.05$

Table 7 Independent t-test of the mean post traumatic stress scores of the younger children (9-I2) and teenagers (I3-I6)

\begin{tabular}{lllll}
\hline PTSD Score & Groups & & & \\
\cline { 2 - 5 } & Younger Children (9-1 2) & Teenagers (13-16) & Mean Difference & t \\
\hline Pre-test & 26.18 & 27.68 & 1.5 & 0.595 \\
Post-test & 25 & 24.52 & 2.3 & 0.556 \\
Mean Difference & 1.18 & 3.1642 & & 0.208 \\
\hline
\end{tabular}

*Statistically significant at $\mathrm{P}<.05$

\section{Comparison between treatment group and control group}

As shown on the Table 5 before treatment the mean of post traumatic stress symptoms score of the treatment group was 27.13 while the mean of post traumatic stress score of the control group was 28 the difference of the means of the two groups being 0.86 . An independent $t$ test of the equality of the two means indicates that the difference of the two means of PTSD score was not significant at 0.05 .

After the treatment the mean post traumatic stress symptoms score of the treatment group was 24.7 and the mean of post traumatic stress score for the control group was 27.56. the difference of the mean for the treatment group and for the control group was 2.3. When a two tailed $t$ test was used to test the mean difference between the control group and the treatment group, there was statistically significant difference between the two means at $0.05(d f=58, t=2.068)$. That is the treatment group showed statistically significant reduction in the mean score of post traumatic stress symptoms at post test compared to the control group.

\section{Comparison of post traumatic stress across type of abuse and age}

As Table 6 shows the mean score of post traumatic stress symptom scale at pre test was 28.07 for sexually abused children and 26.41 for physically abused children. The difference of the mean post traumatic stress symptoms for sexually abused children and for physically abused children was 1.66. The difference between the two means (sexually abused and physically abused) before treatment is not statistically significant at 0.05 . The mean score of PTSD symptoms for sexually abused children was slightly more than that for physically abused even though it was not statistically significant.

It is also indicated on the table that the mean score of PTSD symptoms after the treatment (post test 1 ) was 24.88 for sexually abused children and 24.46 for physically abused children. The difference between the two means was 0.429 which is not statistically significant when tested with independent $t$ test at 0.05 . However the difference was still maintained that sexually abused children scored higher on PTSD symptoms scale once again after the treatment.

As can be seen on Table 7 the mean post traumatic stress symptoms score before treatment for younger children(children aged 9-12) was 26.18 while it was 27.68 for the teenagers (13-16 years of age), the teenagers scoring slightly more than the younger children.
The difference between the mean post traumatic stress score of the two groups was 1.50 which is not statistically significant tested with independent two tailed t test at 0.05 .

When the test was administered after the treatment the mean post traumatic stress symptoms score for teenagers (13-16 years) was (24.52) was less than the younger children (25). The difference of the two means which is 2.30 was not statistically significant when tested with independent $t$ test at 0.05 .

\section{Discussion}

This study was conducted to examine the effectiveness of trauma focused cognitive behavioral group therapy to treat PTSD symptoms of children who experienced sexual or physical abuse. To these end the results of the finding are discussed in the light of the literature.

\section{Post traumatic stress symptoms before the treatment}

The finding of this study indicated that majority of children who are the victims of physical or sexual abuse show moderate level of post traumatic stress as measured by CPSS. As indicated in the results section, $63.4 \%$ of participants in the treatment group showed moderate or severe level of post traumatic stress symptoms $(56.7 \%)$ moderate and $6.7 \%$ sever).Majority of the participants in the control group as well were at moderate $(60 \%)$ and sever $(6.7 \%)$ level of post traumatic stress symptoms.

This finding is consistent with many other findings. Rodriguez et al., ${ }^{13}$ found that $72 \%$ of sexually abused children from clinical sample met criteria for current post traumatic stress disorder. Saunders et al. ${ }^{4}$ found significantly higher rates of lifetime posttraumatic stress symptoms in participants reporting contact sexual abuse and child rape compared to participants reporting non-contact sexual abuse experiences. In a clinical sample up to $90 \%$ showed PTSD symptoms, however, all of these children have experienced trauma other than sexual abuse. ${ }^{14}$

In the study of children and adolescents ( 9 to 16years old) who live in child care centers in North Carolina USA found out that $90 \%$ of them experienced at least one traumatic event in their life time (North Carolina department of social services and the family and childrens' resource program, 2005). In another study in USA researchers examined PTSD symptoms of 659 young adults who had been placed in family foster care found that one in four $(25 \%)$ of them had experienced PTSD which is double of the US war veterans. Most of them were sexually or physically abused..$^{15}$ 
Before the treatment there was little to no difference between the control group and the treatment group, with regard to the socio demographic variables like age, educational level and time stayed in the institutions. There were equal number of sexually abused children and physically abused children in the treatment group as well as in the control group. As far as post traumatic stress symptom is concerned the mean score of the control group was 28 while it was 27.13 for treatment group indicating that there is no statistically significant difference between the two groups.

The study also has shown that the mean post traumatic stress symptom for sexually abused children was 28.07 before treatment and it was 26.41 for physically abused children. The difference between the means is not statistically significant though. The literature confirms that physically and sexually abused children show similar types of symptoms of anxiety, low self esteem, depression and post traumatic stress disorder. ${ }^{2}$

The literature shows that both sexually abused and physically abused children frequently experience PTSD symptoms. The difference between the two groups is the type of symptoms they show. Sexually abused children frequently experience flash backs, repetitive unwanted memories, and abuse related nightmares while physically abused children usually avoid trauma related memories and stimuli, experience violent nightmare and irritability. ${ }^{7}$

As indicated in the results section, the mean PTSD score before treatment was 27.68 for the teenagers while it was 26.1818 for the younger children. The teenagers scored slightly more than the younger children but were not statistically significant.

Literature indicates that there are some differences in the expression of PTSD symptoms between elementary school children and adolescents. Adolescents show more adult like symptoms like flash backs, nightmare ,emotional numbing, avoidance of trauma related stimuli, substance abuse, withdrawal, suicidal thoughts, school avoidance, sleep disturbances and confusion. In younger children extreme withdrawal, inability to concentrate, nightmares, irritability refusal of school, outburst of anger are more common. ${ }^{15}$

\section{Post traumatic Stress symptoms after the Treatment}

The treatment group showed statistically significant change in post traumatic stress symptoms at the second point of measurement (post test one). The mean post traumatic stress symptom score shifted from 27.13 before treatment to 24.7 after treatment which is statistically significant difference. Here it would be appropriate to mention some single design pre-post test studies that examined the effectiveness of cognitive behavioral therapy. In one of such studies, Deblinger et al., ${ }^{16}$ investigated the effectiveness of CBT for children aged 3-16 suffering from PTSD.

The study included 19 children and they were assessed using structured clinical interview. Measures of PTSD and other symptoms were taken twice before treatment (to check for symptom stability) and ones after treatment. The children showed significant improvement in many symptoms and no child meet the criteria for clinical PTSD symptom after treatment.

The control group also showed reduction of post traumatic stress symptoms from the mean of 28 before treatment to the mean of 27.56 after treatment. The difference was 0.44 which is not statistically significant. The insignificant improvement in post traumatic stress symptoms can be explained by some findings which suggest that abused children show improvement in symptoms by the mere passage of time. Some suggested that the time since the last abuse is responsible for the improvement while others stressed the time since the discovery of the abuse is important particularly in the case of sexual abuse. ${ }^{8}$

There are findings which show that in the long run PTSD symptoms get reduced whether therapy is provided or not. For instance, Foa et al. ${ }^{17}$ conducted a non randomized controlled trial on 20 children suffering from PTSD symptoms where he provided short term TF-CBT. Fallow up measure after two months showed that there were fewer cases of PTSD in treatment group than in comparison group. After five months the difference between the two groups was only the severity of the symptoms. ${ }^{18}$

As indicated in the results section, at post test the mean post traumatic stress symptom measure of the treatment group was 24.7 while it was 27.56 for the control group. An independent $t$ test indicates that the difference between the two means is statistically significant. This shows that the therapeutic intervention has brought about significant change among the treatment group compared to a control group.

This finding is consistent with many other findings which show the effectiveness of TF-CBT to treat children with PTSD symptoms. For instance, one study by Maritt et al. ${ }^{18} 143$ patients with acute post traumatic stress disorder was randomly assigned to either brief cognitive behavioral therapy or a comparison waiting list. Cognitive behavioral therapy was consisted of education, relaxation exercise, imaginable exposure, in vivo exposure and cognitive restructuring. Main outcome of PTSD score was measured by structured interview. The outcome was measured just after the intervention and 4 months after the intervention. PTSD symptoms showed significant reduction among treatment group compared to the waiting list just after the treatment and the difference between the two groups was insignificant after 4months.

In other study, Berliner and Saunders ${ }^{19}$ have investigated the effectiveness of Cognitive behavioral group therapy to reduce various symptoms including PTSD, anxiety and depression; they divided 86 children in to treatment group and comparison group. The treatment group received cognitive behavioral group therapy with co-counselors. Outcome scores were measured just after the treatment and one year after the treatment. The result was that the treatment group showed statistically significant improvement of symptoms for the treatment group. ${ }^{18}$

Cloitre and Koenen ${ }^{20}$ recruited participants with posttraumatic stress symptoms related to child sexual abuse to participate in a study of a 12-week interpersonal process group. Participants in the groups in which there were no members diagnosed with borderline personality disorder showed significant improvement on measures of anger and posttraumatic stress symptoms.

After the treatment, the mean of PTSD score for the younger children was 25 while it was 24.52 for adolescents. Here the teenagers scored less than the younger children. Even though, the difference was not significant it might indicate that the teenagers recovered better than the younger children suggesting that they were more responsive to therapy.

Research findings indicated that CBT is comparatively more effective than other therapies for different age groups from preschoolers to adults. For instance, Cohen and Marino ${ }^{21}$ randomly assigned eighty six preschool children either to a cognitive behavioral therapy or to a non directive supportive therapy. Treatment outcome measures included behavior check list, the child sexual behavior inventory and the weekly behavior report. Children who were treated 
with CBT showed significantly better improvement in all measures compared to children who received non directive supportive therapy. They repeated similar design for older children aged 7-14 and used social competence scale, behavior profile scale, children's depression inventory, and sexual behavior inventory. CBT was found to be significantly more effective in older children ( 7 to14years old) as well.

The finding of the study indicates that there was no significant difference in mean score of post traumatic stress symptoms between the children who experienced sexual abuse and children who experienced physical abuse both before and after the treatment. However, sexually abused children showed consistently higher score in both pre and post tests than physically abused children though it was not significant.

The consistent higher score of sexually abused children compared to physically abused children even though not statistically significant can be explained by some literature indicating sever PTSD on sexually abused children than physically abused children. ${ }^{8}$ It can also be due to different symptoms of PTSD experienced by the two groups.

These findings are encouraging that TF-CBGT can help abused children with PTSD recover from the symptoms better than no treatment situation. Further studies are needed to verify and expand these findings in different settings.

\section{Follow up}

Follow up measure of post traumatic stress symptoms was performed after 15 days of the first treatment outcome test. This was in order to test whether the treatment outcome will be maintained through time. The test revealed that post traumatic stress symptoms show slight increase compared to post test one. However, the difference was too small to be statistically significant which indicates that the treatment outcome was maintained at post test 2 .

There are contrasting findings regarding the consistency of treatment outcome at follow up studies. A study by Deblinger et al., ${ }^{16}$ fallowed children with PTSD who receive cognitive behavioral therapy for two years measuring PTSD symptoms, child depression inventory and child behavior checklist. The measures were taken 1 month, 6month, 12 month and 2 years after the treatment program. The result shows that the treatment gain was maintained in all the tests and in all follow up times.

In another study, Marit et al., ${ }^{18}$ have shown that the participants in short term TF-CBT treatment program recovered from PTSD symptoms one week after the intervention. It was conducted on a sample of 143 which is divided in to treatment group (79) and control group (64). Four months after intervention the difference between the control group and the treatment group in symptoms of PTSD, depression and anxiety was insignificant.

The amount of evidence that show the effectiveness of trauma focused cognitive behavioral therapy is growing worldwide and this research adds to the growing evidence. The strength of theses study is the presence of a control group with which to compare the outcome measures. However, it was not intended to be a randomized controlled study where the two groups are equivalent.

This study included a limited number and variety (only 30 children living in charity organizations) of participants which limits its applicability to the general population. It would have been better if the fallow up test was long after the treatment and repeated measure in order to test whether the treatment outcome is maintained. Future studies may examine the effectiveness of TF-CBT on larger sample and with a repeated fallow up over a long period of time in order to test the consistency of results over time.

\section{Conclusion}

In this study majority of the participant's experienced moderate levels of post traumatic stress disorder symptoms before the treatment. TF-CBGT is shown to reduce post traumatic stress symptoms among treatment group and the treatment gains were maintained in follow up measure. The treatment is effective for both sexually abused and physically abused children. It is also shown that both younger children and adolescents are equally responsive to the treatment.

This study has indicated that trauma focused cognitive behavioral therapy can be effective in reducing post traumatic stress symptoms of both sexually and physically abused children. However, we need extensive large scale studies with larger and more diverse samples before we can talk about TF-CBT in Ethiopian context with adequate certainty. Given the number of children suffering from various types of abuse and traumas that actually need psychological and mental health services together with this kind of study make it necessary to conduct more studies on the effectiveness and adaptation of various psychotherapies particularly TF-CBT.

\section{Author's contribution}

Riyadh Mohammed, contributed in problem identification, first proposal preparation and presentation, conducting intervention, data collection, preformed statistical analysis and final report writing and presentation. Daniel Sewasew advised in intervention planning, method section; study design and analysis part, and drafting and editing the manuscript. Gebeyehu Abate consulted in proposal part, method section; study design and analysis part and edit the final written report. Mastewal Bitew contributed in reading and editing the manuscript, data collection and analysis part.

\section{Acknowledgements}

First and for most, we express our gratitude to Mr. Moges Ayele for his constructive and valuable comments and suggestions. Second, we really appreciate genuine cooperation of counselors and social workers in Kechene children's home and OPRIFS. Finally, we deeply indebted to those who were involved as participants, for their participation and collaboration in this study.

\section{Conflicts of interest}

Author declares there are no conflicts of interest.

\section{Funding}

None.

\section{References}

1. World Health Organization, WG. World Report on Violence and Health 2002. p.82-111.

2. Carr A. Family Therapy Concepts, Process and Practice. ( $2^{\text {nd }}$ edn), Chichester: John Welly \& Sons Ltd, New York, USA. 2006.

3. World Health Organization. Report on the Consultation on Child Abuse Prevention. 2004. p.1-60.

4. Saunders BE, Berliner L, Hanson RF. Child Physical and Sexual Abuse: Guidelines for Treatment. National Crime Victims Research and Treatment Center, Charleston, South Carolina, USA. 2004. p. 1-137.

5. Glaser D. Treatment Issues in Child Sexual Abuse. Br J Psychiatry. 1991;159:769-782.

6. Hamblen J . PTSD in Children and Adolescents. 2003. 
7. Briere J. Child Abuse Trauma: Theory and Treatment of the Lasting Effects. Newbury Park, CA: Sage Publications, USA. 1992.

8. Skowron EA, Reinemann DHS. Psychological interventions for child maltreatment: A meta- analysis. Psychotherapy: Theory, Research, Practice, and Training. 2005;42(1):52-71.

9. King NJ, Tonge BJ, Mullen P, et al. Cognitive- behavioral treatment of sexually abused children: a review of research. Behavioral and Cognitive Psychotherapy . 1999;27(4):295-309.

10. Gobena D. Child sexual abuse in Addis Ababa high schools. 1998.

11. National Child Traumatic Stress Network. How to Implement TraumaFocused Cognitive Behavioral Therapy (TF-CBT). National Child Traumatic Stress Network (NCTSN). 2008.

12. Foa EB, Johnson KM, Feeny NC, et al. The Child Posttraumatic Symptom Scale: A Preliminary Examination of its Psychometric Properties. J Clin Child Psychol. 2001;30(3):376-384.

13. Rodriguez N, Ryan SW, Foy DW. Tension reduction and PTSD Survivors of sexual abuse. Paper presented at the annual meeting of the International Society for Traumatic Stress Studies, Los Angeles, USA. 1992.

14. Finkelhor D, Berliner L. Research on the treatment of sexually abused children: A review and recommendations. J Am Acad Child Adolesc Psychiatry. 1995;34(11):1408-1423.
15. North Carolina department of social services and the family and childrens' resource program. Children's service practice Notes. 2005.

16. Deblinger E, Steer RA, Lippmann J. Two-year follow-up study of cognitive behavioral therapy for sexually abused children suffering posttraumatic stress symptoms. Child Abuse Negl. 1999;23(12):1371-1378.

17. Foa EB, Riggs DS, Dancu CV,et al. Reliability and validity of a brief instrument for assessing post-traumatic stress disorder. Journal of Traumatic Stress. 1993;6(4):459-473.

18. Sijbrandij M, Olff M, Reitsma JB, et al. Treatment of Acute Posttraumatic Stress Disorder With Brief Cognitive behavioral therapy. Am J Psychiatry. 2007; 164(1):82-90.

19. Berliner L, Saunders BE. Treating fear and anxiety in sexually abused children: results of a controlled 2-year follow-up study. Child Maltreat. 1996;1(4):294-309.

20. Cloitre M, Koenen KC. The impact of borderline personality disorder on process group outcome among women with posttraumatic stress disorder related to childhood abuse. Int J Group Psychother. 2001;51(3):379-398.

21. Cohen JA, Mannarino AP. A treatment outcome study for sexually abused preschool children: initial findings. J Am Acad Child Adolesc Psychiatry. 1996;35(1):42-50. 